\title{
Auch Arztdaten werden künftig erfasst
}

\author{
Der Bundestag hat grünes Licht für das umstrittene Gesetz zur Vorrats- \\ datenspeicherung gegeben. Das nimmt Geheimnisträger wie Ärzte \\ nicht von der Datenerfassung aus.
}

$\mathrm{K}$ ünftig sollen Telekommunikationsunternehmen und Internetprovider Verbindungsdaten zu Telefongesprächen, IP-Adressen von Computern sowie Datum, Uhrzeit und Dauer der Verbindungen speichern. So sieht es das im Eilverfahren vom Bundestag beschlossene Gesetz zur Vorratsdatenspeicherung vor.

Die Telefon- und Verkehrsdaten müssen dabei acht Wochen, die MobilStandortdaten vier Wochen vorgehalten werden. Kommunikationsinhalte sollen nicht erfasst werden. Nach Recherchen von Datenschützern aus dem Arbeitskreis Vorratsdatenspeicherung speichern Telekommunikationsfirmen allerdings üblicherweise auch die Inhalte von SMS. Die Aktivisten befürchten einen Missbrauch dieser Daten.

Problematisch für Ärzte ist vor allem, dass das Gesetz die Geheimnisträger nicht von der Datenspeicherung ausnimmt. Die Strafprozessordnung regelt in Paragraf 100g Abs. 4 zwar, dass die Erhebung der Daten von Geheimnisträgern, über die diese das Zeugnis verweigern dürfen, unzulässig ist - sie also nicht verwertet werden dürfen. Gleichzeitig nimmt der Gesetzgeber im nun verabschiedeten Vorratsdatengesetz jedoch nur Personen, Behörden und Organisationen in sozialen oder kirchlichen Bereichen, die anonyme Beratung anbieten, von der Datenspeicherung aus. Die Daten der übrigen Geheimnisträger lassen sich jedoch nicht vorab herausfiltern. Es zeigt sich erst beim Zugriff, ob jemand Arzt, Jurist oder Tatverdächtiger ist. Ärzte- und Zahnärzteschaft, Apotheker,
Rechtsanwälte, Steuerberater und Wirtschaftsprüfer hatten sich noch vor dem Beschluss daher gemeinsam gegen die anlasslose flächendeckende Speicherpflicht von Berufsgeheimnisträgern ausgesprochen.

\section{Nach drei Jahren Evaluation geplant}

Die einzige wichtige Änderung, die in den Entwurf der Bundesregierung noch eingebaut wurde, ist, dass nach drei Jahren eine Evaluation des Gesetzes erfolgen muss. Der Digitalverband Bitkom übt harsche Kritik: „Es ist fraglich, ob die angestrebten Ermittlungserfolge einen derart starken Eingriff in die Grundrechte der Bürger rechtfertigen“, so BitkomHauptgeschäftsführer Dr. Bernhard Rohleder. Laut dem Verband hätte es vor allem auch bei der Umsetzung einer intensiveren Diskussion benötigt. „Die Telekommunikationswirtschaft muss die gesetzlichen Vorgaben umsetzen, wurde zur praktischen Ausgestaltung des Gesetzes aber gar nicht gefragt", so Rohleder. Noch sei unklar, wie eine solche Vorgabe umzusetzen ist. Rohleder rechnet damit, dass das Gesetz „mit hoher Wahrscheinlichkeit wieder vor dem Verfassungsgericht landen wird“. Der Verband schätzt zudem, dass die Umsetzung die Unternehmen einen mittleren dreistelligen Millionenbetrag kosten wird. Und auch die Bundesdatenschutzbeauftragte Andrea Voßhoff sagt: „,Meine grundsätzlichen verfassungsrechtlichen Bedenken bleiben bestehen." Mehrere Politiker und Initiativen kündigten bereits an, gegen das Gesetz zu klagen.

RebekkaHöhl, 